\title{
Analyzing power in elastic scattering of the electrons off a spin-0 target
}

\author{
Mikhail Gorchtein* and Charles J. Horowitz ${ }^{\star}$ \\ Nuclear Theory Center and Department of Physics, \\ Indiana University, Bloomington, IN 47408, USA
}

(Dated: November 2, 2018)

\begin{abstract}
We consider the analyzing power on a spin-0 nuclear target. This observable is related to the imaginary part of the two-photon-exchange (box) diagram. We consider the contributions of elastic and inelastic intermediate states. The former requires knowledge of the elastic nuclear form factor, while the latter uses the optical theorem as input. The elastic contribution scales as the nuclear charge $Z$, while the inelastic contribution as the ratio of the atomic number and nuclear charge, $A / Z$. We provide estimates for ${ }^{4} \mathrm{He}$ and ${ }^{208} \mathrm{~Pb}$, in the kinematics of existing or upcoming experiments. In both cases, we predict negative values for the analyzing power of a few parts per million, and the dominant contribution is due to inelastic intermediate states. The analyzing power can contribute a substantial systematic error in parity-violating experiments.
\end{abstract}

PACS numbers: 21.10.Pt, 25.30.Bf, 25.30.Fj, 27.10.+h, 27.80.+w

\section{INTRODUCTION}

Over the past few years, much attention was paid to the two-photon-exchange (TPE) effects in elastic electron scattering off nucleons and nuclei. The discrepancy between the values of the elastic form factor ratio of the proton, $G_{E} / G_{M}$ obtained with the Rosenbluth separation technique 2 on one hand, and the polarization transfer technique 1] on the other hand, is believed to be due to these TPE effects [3]. To ultimately disentangle these effects, two experiments are planned at JLab [4] and at VEPP ring [5] that will measure the ratio of the electron and positron cross sections.

Another way to measure the TPE effects is to study the analyzing power, called Mott asymmetry in low energy polarimetry. This asymmetry involves a transversely polarized beam of electrons. Because of time reversal symmetry, a non-zero asymmetry requires a non-zero imaginary part of the elastic amplitude and is due to exchange of at least two photons. This observable scales naively as $\frac{m_{e}}{E} Z \alpha_{e m}$, with $m_{e}$ the electron mass, $E$ the beam energy, $Z$ the charge of the target particle, and $\alpha_{e m}$ the fine structure constant. A rough estimate gives $10 \mathrm{ppm}$ for the case of $500 \mathrm{MeV}$ beam scattering off the proton target.

Parity violating experiments use a longitudinally polarized beam of electrons and measure the difference in cross section due to flipping the beam polarization. Such $\mathrm{PV}$ asymmetries are typically of order $1 \mathrm{ppm}$. It can be seen, that a small transverse component of the electron spin can lead to a substantial systematical effect on the $\mathrm{PV}$ asymmetry. On the other hand, the analyzing power can be measured easily with the same apparatus used in PV experiments. There exist several measurements of this effect [6] and a number of theoretical estimates [7], 8]

*Electronic address: gorshtey@caltech.edu

${ }^{\dagger}$ Electronic address: horowit@indiana.edu for the proton target. In the case of a nuclear target, the effects of the exchange of two photons is expected to be even more important, as it grows with the nuclear charge $Z$. This paper is dedicated to calculating the analyzing power on two spin-0 nuclear targets used in two PV experiments runnning at JLab. HAPPEX experiment 9] uses the ${ }^{4} \mathrm{He}$ target and $3 \mathrm{GeV}$ electron beam. PREX experiment [10] uses the ${ }^{208} \mathrm{~Pb}$ target and $850 \mathrm{MeV}$ electrons. The paper is organized as follows. We start with defining the kinematics and conventions in Section III In Section IIII, we calculate the imaginary part of the elastic electron-nucleus amplitude due to elastic and inelastic intermediate states. In Section IV, we present the results of our calculation for ${ }^{4} \mathrm{He}$ and ${ }^{208} \mathrm{~Pb}$, and discuss their implications for the experiment.

\section{KINEMATICS AND OBSERVABLES}

Kinematics of elastic electron-nucleus scattering process $e(k)+N(p) \rightarrow e\left(k^{\prime}\right)+N\left(p^{\prime}\right)$ is fixed by three independent vectors,

$$
\begin{aligned}
P & =\frac{p+p^{\prime}}{2} \\
K & =\frac{k+k^{\prime}}{2} \\
q & =k-k^{\prime}=p^{\prime}-p,
\end{aligned}
$$

and two independent Mandelstam invariants $Q^{2}=-q^{2}>$ 0 and $\nu=(P \cdot K) / M$, where $M$ denotes the mass of the nucleus. The usual polarization parameter $\varepsilon$ of the virtual photon can be related to the invariants $\nu$ and $Q^{2}$ (neglecting the mass of the electron here):

$$
\varepsilon=\frac{\nu^{2}-M^{2} \tau(1+\tau)}{\nu^{2}+M^{2} \tau(1+\tau)}
$$


with $\tau=Q^{2} /\left(4 M^{2}\right)$. Elastic scattering of electrons off a spin-less nuclei is described by two amplitudes,

$$
T=\frac{e^{2}}{Q^{2}} \bar{u}\left(k^{\prime}\right)\left\{m_{e} A_{1}+A_{2} P\right\} u(k)
$$

The amplitudes $A_{1,2}$ are functions of the invariants $\nu, Q^{2}$. In the one-photon exchange (OPE) approximation, the helicity-flip amplitude $A_{1}$ vanishes, while the amplitude $A_{2}$ is related to the elastic nuclear form factor that only depends on $t$ :

$$
A_{2}^{(0)}=2 Z F_{N}\left(Q^{2}\right)
$$

with $Z$ the nuclear charge. The unpolarized cross section is given by

$$
\frac{d \sigma}{d \Omega_{L a b}}=F_{N}^{2}\left(Q^{2}\right) \frac{d \sigma_{0}}{d \Omega_{L a b}}
$$

with the usual Rutherford cross section

$$
\frac{d \sigma_{0}}{d \Omega_{L a b}}=\frac{4 \alpha^{2} Z^{2} \cos ^{2} \frac{\Theta}{2}}{Q^{4}} \frac{E^{\prime 3}}{E}
$$

$\Theta$ the electron Lab scattering angle and $E\left(E^{\prime}\right)$ the incoming (outgoing) electron Lab energy. The analyzing power, or beam normal spin asymmetry is defined as

$$
A_{n}=\frac{\sigma_{\uparrow}-\sigma_{\downarrow}}{\sigma_{\uparrow}+\sigma_{\downarrow}}
$$

where $\sigma_{\uparrow}\left(\sigma_{\downarrow}\right)$ denotes the respective elastic cross section with the incoming electrons polarized along the positive (negative) normal vector $S^{\gamma}$,

$$
S^{\gamma}=\varepsilon_{\alpha \beta \gamma \delta} P^{\alpha} K^{\beta} q^{\delta}
$$

This observable requires a non-zero imaginary part of the elastic amplitude, thus it is identically zero in the OPE approximation. Including the exchange of two photons, we obtain to leading order in $\alpha_{e m}$

$$
A_{n}=-\frac{m_{e}}{\sqrt{s}} \tan \left(\frac{\theta_{c m}}{2}\right) \frac{\operatorname{Im} A_{1}}{Z F_{N}\left(Q^{2}\right)},
$$

with $\operatorname{Im} A_{1} \sim O\left(\alpha_{e m}\right)$.

\section{IMAGINARY PART OF THE TPE AMPLTUDE}

The imaginary part of TPE amplitude is given by

$$
\operatorname{Im} T_{2 \gamma}=e^{4} \frac{1}{(2 \pi)^{3}} \int \frac{d^{3} \vec{k}_{1}}{2 E_{1}} \frac{1}{Q_{1}^{2} Q_{2}^{2}} l_{\mu \nu} \cdot W^{\mu \nu},
$$

where we explicitly set the intermediate electron on-shell, $E_{1}=\sqrt{\vec{k}_{1}^{2}+m_{e}^{2}}$. The leptonic tensor is given by

$$
l_{\mu \nu}=\bar{u}\left(k^{\prime}\right) \gamma_{\nu}\left(k_{1}+m_{e}\right) \gamma_{\mu} u(k) .
$$

\section{A. Elastic contribution}

In the case of the elastic intermediate state (cf. Fig. 1 ), the hadronic tensor is

$$
\begin{aligned}
W^{\mu \nu} & =\pi \delta\left(\left(P+K-k_{1}\right)^{2}-M^{2}\right) \\
& \times\left(2 p+q_{1}\right)^{\mu}\left(2 p^{\prime}+q_{2}\right)^{\nu} Z^{2} F_{N}\left(Q_{1}^{2}\right) F_{N}\left(Q_{2}^{2}\right)
\end{aligned}
$$

Above, $q_{1}^{\mu}=k-k_{1}$ denote the incoming and $q_{2}^{\mu}=$ $k^{\prime}-k_{1}$ the outgoing photon momenta, and $Q_{1,2}^{2}=-q_{1,2}^{2}$, respectively. Gauge invariance of the leptonic tensor leads to $q_{1}^{\mu} l_{\mu \nu}=q_{2}^{\nu} l_{\mu \nu}=0$. For the imaginary part, the form factors $F_{N}$ are the on-shell form factors, and we will use experimental fits for them. Evaluating the

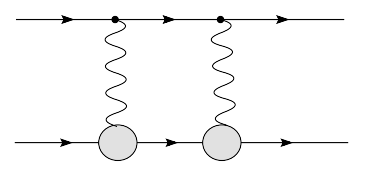

FIG. 1: The nucleus box-graph. The shaded blobs represent the nuclear form factor

remaining $\delta$-function in the c.m. frame, we are left with the integral over electorn's solid angle $\Omega_{1}$,

$$
\operatorname{Im} T_{2 \gamma}^{e l}=\frac{Z^{2} e^{4}}{8 \pi^{2}} \frac{E_{1}}{\sqrt{s}} \int \frac{d \Omega_{1}}{Q_{1}^{2} Q_{2}^{2}} l_{\mu \nu} p^{\mu} p^{\prime \nu} F_{N}\left(Q_{1}^{2}\right) F_{N}\left(Q_{2}^{2}\right),
$$

with the invariant $s=(P+K)^{2}=M^{2}+2 M \nu+Q^{2} / 2$, and $E_{1}=\frac{s-w^{2}}{2 \sqrt{s}}$ denoting the c.m. energy of the intermediate electron. $w^{2}$ stands for the invariant mass squared of the intermediate hadronic state. It equals to $M^{2}$ for the elastic, and lies between the threshold for pion production $\left(M+m_{\pi}\right)^{2}$ and the full energy $s$ for inelastic intermediate states.

The integral over the intermediate electron's solid angles can be rewritten in terms of the exchanged photons' virtualities $Q_{1,2}^{2}$ :

$$
\int d \Omega_{1}=\frac{1}{E E_{1}} \int_{0}^{4 E E_{1}} d Q_{1}^{2} \int_{Q_{-}}^{Q_{+}} \frac{d Q_{2}^{2}}{\sqrt{\left(Q_{+}-Q_{2}^{2}\right)\left(Q_{2}^{2}-Q_{-}\right)}}(14)
$$

The limits of the integration $Q_{ \pm}$are given by

$$
\begin{aligned}
Q_{ \pm} & =\frac{E_{1}}{E} Q^{2}+Q_{1}^{2}-\frac{Q^{2} Q_{1}^{2}}{2 E^{2}} \\
& \pm 2 \sqrt{Q^{2} Q_{1}^{2}} \sqrt{\frac{E_{1}}{E}\left(1-\frac{Q^{2}}{4 E^{2}}\right)\left(1-\frac{Q_{1}^{2}}{4 E E_{1}}\right)}
\end{aligned}
$$

Fig. 2 displays the area of the accessible values of $Q_{1,2}^{2}$ for different kinematics and for the case of the nucleon target. The upper panels display the case of the elastic intermediate state, while the lower panel show the inelastic case for two specific values of $w^{2}$. By means of standard methods including Dirac algebra and the reduction of vector 4-point integrals to scalar 3- and 4-point 

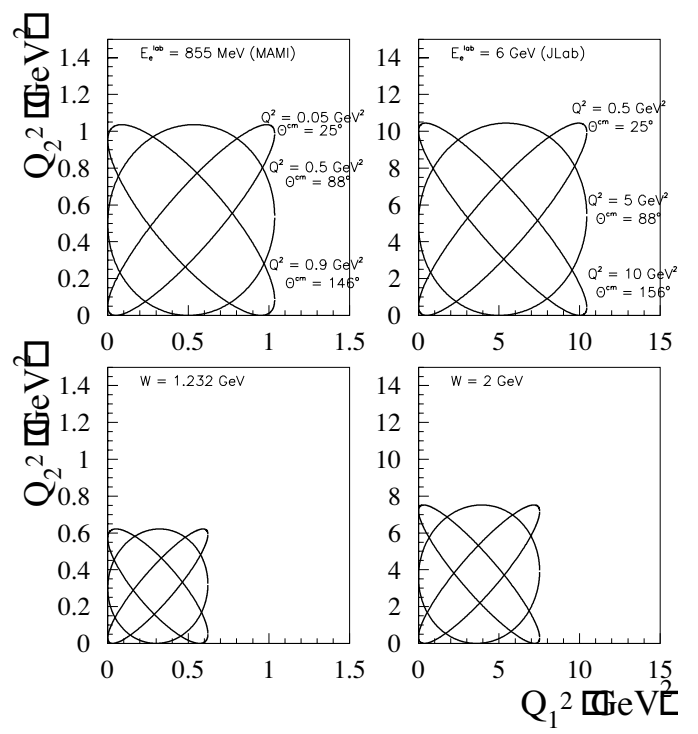

FIG. 2: Allowed values of the exchanged photon virtualities $Q_{1,2}^{2}$ are restricted to be inside the ellipses.

integrals, we can identify the imaginary part of the amplitude $A_{1}$

$$
\begin{aligned}
\operatorname{Im} A_{1}^{e l} & =\frac{Z^{2} \alpha}{\pi} \frac{Q^{2}}{Q^{2}-\frac{\left(s-M^{2}\right)^{2}}{s}} \frac{s+M^{2}}{s-M^{2}} \\
& \times \int_{0}^{4 E^{2}} d Q_{1}^{2} \int_{Q_{-}}^{Q_{+}} \frac{d Q_{2}^{2}}{\sqrt{\left(Q_{+}-Q_{2}^{2}\right)\left(Q_{2}^{2}-Q_{-}\right)}} \\
& \times \frac{Q^{2}-Q_{1}^{2}-Q_{2}^{2}}{2 Q_{1}^{2} Q_{2}^{2}} F_{N}\left(Q_{1}^{2}\right) F_{N}\left(Q_{2}^{2}\right)
\end{aligned}
$$

We notice that the analyzing power does not contain any IR divergencies, so that the integrand in the above formula is regular for any allowed values of $Q_{1,2}^{2}$.

\section{B. Inelastic contribution}

We will next estimate the contribution of the inelastic intermediate states to the imaginary part of $A_{1}$ in the case of forward scattering angles. We can provide a realistic estimate for the case of nearly forward scattering, as it was proposed for the proton target in Refs. [11], [12]. In the forward direction, the imaginary part of the doubly virtual Compton scattering amplitude is given in terms of the structure functions $W_{1,2}$, and making use of Callan-Gross relation, we have

$$
\begin{aligned}
W^{\mu \nu} & =\pi W_{1}\left(w^{2}, Q_{1}^{2}\right) \\
& \times\left\{-g^{\mu \nu}+\frac{P^{\mu} q_{1}^{\nu}+P^{\nu} q_{2}^{\mu}}{(P \tilde{K})}-\frac{\left(q_{1} q_{2}\right)}{(P \tilde{K})^{2}} P^{\mu} P^{\nu}\right\}
\end{aligned}
$$

The structure function $W_{1}$ is related to the virtual photon cross section,

$$
W_{1}=\frac{w^{2}-M^{2}}{2 \pi e^{2}} \sigma_{\gamma^{*} N}\left(w^{2}, Q_{1}^{2}\right)
$$

In Ref. [12], it was shown that for the analyzing power at very forward angles, the $Q_{1,2}^{2}$ dependence of the cross section can be neglected, as it leads to corrections in powers of $Q^{2} / k^{2}$, thus

$$
W_{1} \approx \frac{w^{2}-M^{2}}{2 \pi e^{2}} \sigma_{\gamma N}\left(w^{2}\right)
$$

The integral over the electron's angles can be performed analytically, and we are left with the integral over the lab photon energy $\omega=\frac{w^{2}-M^{2}}{2 M}$ :

$$
\begin{aligned}
\operatorname{Im} A_{1}^{\text {inel }}=\frac{1}{4 \pi^{2}} \frac{M}{E_{l a b}} & \int_{0}^{E_{l a b}} d \omega \omega \sigma_{\gamma N}(\omega) \\
& \times \ln \left[\frac{Q^{2}}{m^{2}}\left(\frac{E_{l a b}}{\omega}-1\right)^{2}\right],
\end{aligned}
$$

with $E_{l a b}=\frac{s-M^{2}}{2 M}$ is the lab electron energy. Photoabsorption cross section has been measured from threshold to high energies for various nuclei, and it is known to approximately scale as the atomic number of the nucleus. Therefore, the inelastic contribution to $A_{n}$ scales approximately as $\frac{A}{Z}$, while it scales as $Z$ for the elastic contribution.

\section{RESULTS AND DISCUSSION}

We now present our results for the analyzing power. We combine Eqs.(16) with Eq.(9) for the contribution of the elastic intermediate state. For the inelastic contribution, additional input is required. We have calculated the imaginary part of the amplitude $A_{1}$ by taking the exact forward limit for the nuclear Compton amplitude where the optical theorem is applicable. To depart from zero scattering angle, we have to make an assumption about the $t$-dependence of Compton scattering amplitude. In the case of the proton, Refs. [11] and [12] use the slope of the differential Compton cross section for the proton target [13] known for $-t=Q^{2} \leq 1 \mathrm{GeV}^{2}$,

$$
\frac{d \sigma}{d t} \approx\left[\frac{d \sigma}{d t}\right]_{t=0} \times e^{B t}
$$

with $B \approx 8 \mathrm{GeV}^{-2}$. Since the differential cross section is related to the amplitude squared, the $t$-dependence is naturally modelled by

$$
\operatorname{Im} A_{1}\left(\nu, Q^{2}\right) \approx \operatorname{Im} A_{1}(\nu) \times e^{-B Q^{2} / 2}
$$


Generalizing this approach to the case of the nuclear target, we can write for the analyzing power:

$$
\begin{aligned}
A_{n}^{\text {inelast }} & \approx-\frac{1}{4 \pi^{2}} \frac{m_{e}}{E_{l a b}} \frac{M}{\sqrt{s}} \frac{A}{Z} \frac{g_{N}\left(Q^{2}\right)}{F_{N}\left(Q^{2}\right)} \tan \frac{\theta_{c . m}}{2} \\
& \times \int_{0}^{E_{l a b}} d \omega \omega \sigma_{\gamma p}(\omega) \ln \left[\frac{Q^{2}}{m^{2}}\left(\frac{E_{l a b}}{\omega}-1\right)^{2}\right]
\end{aligned}
$$

where $g_{N}\left(Q^{2}\right)$ is the phenomenological Compton form factor for a nucleus $N$, and we made use of an approximate scaling of the photo absorption cross section with the atomic number $A$. Unfortunately, the $t$-dependence of Compton data is not known for nuclei. Since the $t$ dependence of the elastic form factors of nuclei is much steeper than that of the nucleon, we also expect that the slope of Compton differential cross section should be much steeper, as well. Therefore, to provide an adequate prediction for the inelastic states contribution to the analyzing power at a non-zero scattering angle, we make a substitution in the above formula:

$$
\frac{g_{N}\left(Q^{2}\right)}{F_{N}\left(Q^{2}\right)} \rightarrow \frac{g_{p}\left(Q^{2}\right)}{F_{1}^{p}\left(Q^{2}\right)}
$$

with $g_{p}\left(Q^{2}\right)=\operatorname{Exp}\left[-\frac{B}{2} Q^{2}\right]$ and $F_{1}^{p}$ the proton Dirac form factor.

If we assume that the photoabsorption cross section is a constant in energy (which is roughly the case at energies above the resonance region, say, $\omega \geq 2.5 \mathrm{GeV}$, with $\sigma_{\gamma p} \approx 0.1$ mbarn), the integration can be performed analytically, and we obtain a simple formula

$$
A_{n}^{\text {inelast }} \approx A_{n}^{0} \frac{g_{N}\left(Q^{2}\right)}{F_{N}\left(Q^{2}\right)} \tan \frac{\theta_{c . m .}}{2}\left(\ln \frac{Q^{2}}{m^{2}}-2\right)
$$

where $A_{N}^{0}=-\frac{m_{e} E_{l a b} \sigma_{\gamma p}}{8 \pi^{2}} \frac{M}{\sqrt{s}} \frac{A}{Z} \approx-4 \mathrm{ppm}$ for lead. This result is analogous to that of Refs. [11] and [12] obtained for the spin- $\frac{1}{2}$ target. Analyzing this formula for a heavy nucleus, we can deduce the energy dependence at very forward angles, where the Compton slope is irrelevant:

$$
A_{n} \sim A_{n}^{0} \tan \frac{\theta_{c . m .}}{2}\left(\ln \frac{4 E_{l a b}^{2}}{m_{e}^{2}}-2+2 \ln \sin \frac{\theta_{c . m .}}{2}\right)
$$

Having in mind that $A_{n}^{0}$ defined above grows linearly with energy, we see that at fixed (forward) angle, the analyzing power behaves as $E \ln E$. At high energies, the phenomenological $t$-dependence tends to partially cancel this growth. On the other hand, for fixed momentum transfer, the analyzing power is practically independent on the beam energy, as was noticed in [11.

We present the results for the $A_{n}$ for two different spin0 nuclei. In Fig. 3, we display the analyzing power on ${ }^{208} \mathrm{~Pb}$ in the kinematics of the PREX experiment, 850 $\mathrm{MeV}$ beam and forward angles. It can be seen that the inelastic contributions give the main contribution, although the elastic contribution is also not negligible. The sum of the two leads to approximately $-4 p p m$ at 6 degrees. The elastic curve in Fig. 3 corresponds not to the calculation presented in this paper, but to the calculation of Ref. [14] that sums the Coulomb distortion effects to all orders in $Z \alpha_{e m}$. In Fig. 4, we display the inleastic contribution to

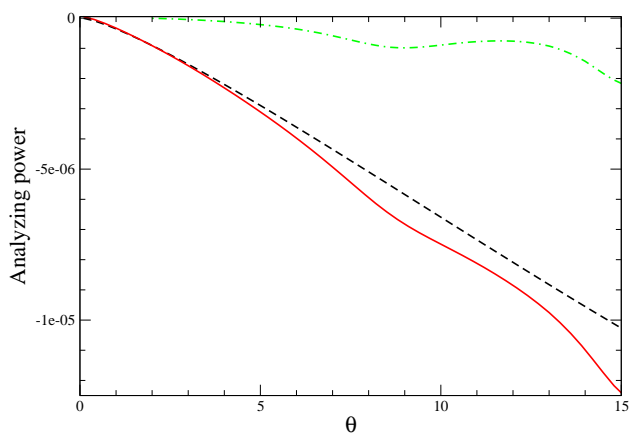

FIG. 3: Analyzing power on ${ }^{208} \mathrm{~Pb}$ at the electron beam energy of $850 \mathrm{MeV}$ as function of the c.m. scattering angle in degrees. Contributions from elastic (dash-dotted) and inelastic (dashed) intermediate states are shown, as well as their sum (solid).

$A_{n}$ on lead at forward angles and a higher energy of 2.7 $\mathrm{GeV}$. The elastic contribution is not shown, as it is very small in those kinematics. For the ${ }^{4} \mathrm{He}$ target, the elastic

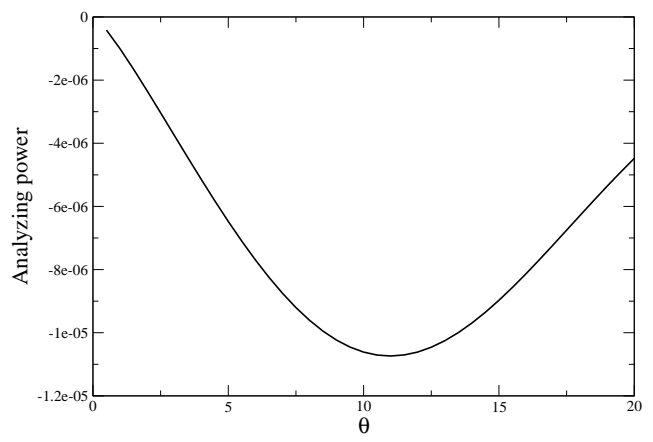

FIG. 4: Inelastic contribution to analyzing power on ${ }^{208} \mathrm{~Pb}$ at the electron beam energy of $2.7 \mathrm{GeV}$ as function of the c.m. scattering angle in degrees.

contribution is largely suppressed, both by a smaller nuclear charge than in the case of lead and by kinematics. The only sizeable contribution comes from the inelastic intermediate states and accounts for about $-10 \mathrm{ppm}$ at 10 degrees c.m. scattering angle. This result closely agrees with the calculation of Ref. [15]. The exact number has to be taken with care, as it relies on a model-dependent $t$-slope that was taken the same as for the proton. This model should work at very small values of $Q^{2}$, but will fail at larger values. Whether or not the point $Q^{2} \approx 0.1$ $\mathrm{GeV}^{2}$ is inside this reliable range, is definitely worth a 
future study. Finally, we discuss the quality of the lead-

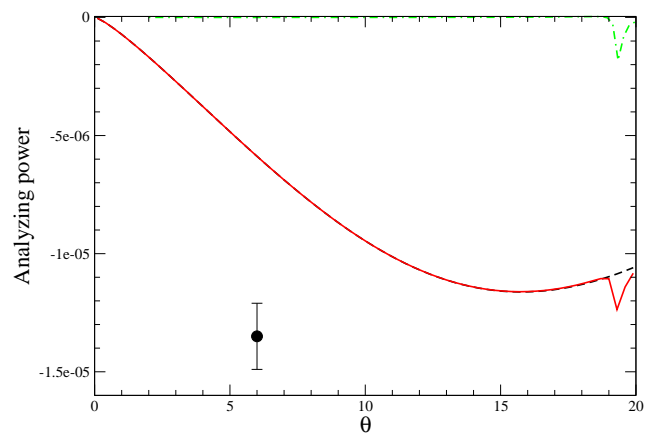

FIG. 5: Analyzing power on ${ }^{4} \mathrm{He}$ at the electron beam energy of $3 \mathrm{GeV}$ as function of the c.m. scattering angle in degrees. Contributions from elastic (dash-dotted) and inelastic (dashed) intermediate states are shown along with the sum of the two (solid). The data point is from [9].

ing order in $\alpha_{e m}$ approximative result for the analyzing power of Eq.(16) by comparing it to the full result of Ref.[14]. This comparison is shown in Figs. 6 and 8 for ${ }^{4} \mathrm{He}$ and ${ }^{208} \mathrm{~Pb}$ target, respectively. The expansion is performed in "small" parameter $Z \alpha_{e m}$, thus it is expected to work well for helium, but not for lead where $Z \alpha_{e m} \approx 0.6$. Indeed, Figs. 6 and 7 demonstrate that for the whole interval in the scattering angle, the agreement between the two calculations is good, apart from the vicinity of the diffraction minimum in the ${ }^{4} \mathrm{He}$ elastic form factor that enters the denominator of Eq.(16). The leading order form factor is exactly zero in the diffraction minimum, while this minimum is partially filled by including Coulomb distortion effects in Ref.[14]. For

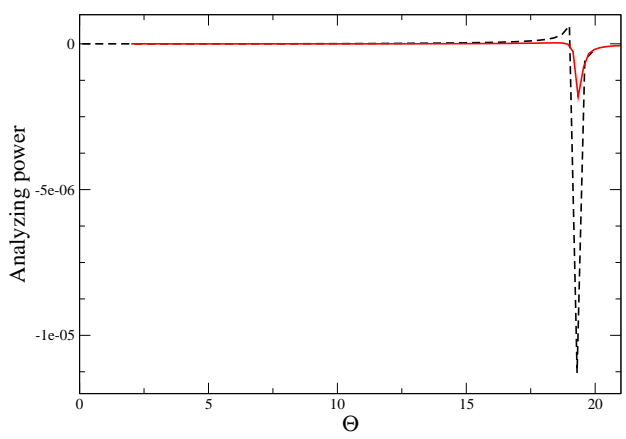

FIG. 6: Elastic contribution to the analyzing power on ${ }^{4} \mathrm{He}$ at the electron beam energy of $3 \mathrm{GeV}$ as function of the c.m. scattering angle in degrees. The leading order contribution (dashed curve) is compared to the full result (full curve) from Ref. [14].

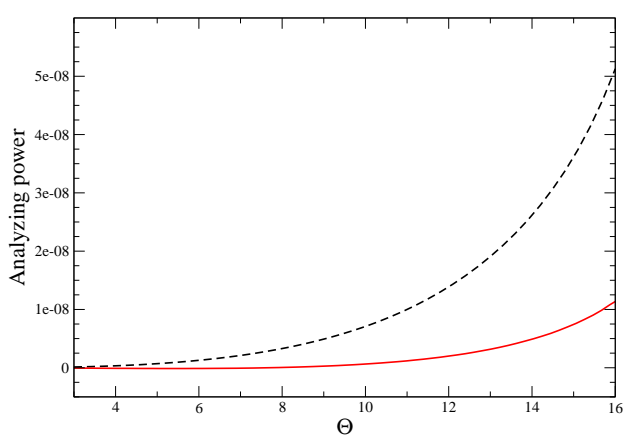

FIG. 7: Zoomed version of Fig. 6]

lead, the agreement between the two calculations is unsatisfactory, and the elastic contribution to the analyzing power is relatively large, so it is necessary to include the higher orders, as well. We quote some of our numerical

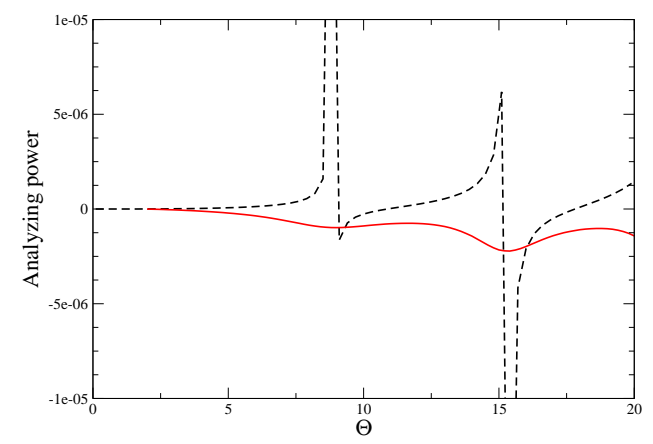

FIG. 8: The same as in Fig. 6 for the case of ${ }^{208} \mathrm{~Pb}$.

results in the kinematics of HAPPEX and PREX expreriments in Table [.

In summary, we considered elastic scattering of electrons off the spin- 0 nuclear target. The analyzing power for this scattering process is related to the imaginary part of the scattering amplitude, and thus requires an exchange of at least two photons. On one hand, the elastic intermediate state contribution is due to Coulomb distortion and can be calculated to all orders in the electromagnetic coupling constant [14]. Another approach capitalizes on the fact that the imaginary part of the forward Compton amplitude is related by the optical theorem to the total photo absorption cross section. Photoabsorption was measured on many nuclear targets, and we use it as input along with the $t$-dependence of the differential Compton cross section which is needed in order to depart from the exact forward limit. We applied this approach to ${ }^{4} \mathrm{He}$ and ${ }^{208} \mathrm{~Pb}$ nuclei in the kinematics of present parity-violation experiments and found that the 


\begin{tabular}{|l|l|l|l|l|l|}
\hline$\Theta_{c . m .}(\mathrm{deg})$ & \multicolumn{2}{|c|}{$A_{n}(\mathrm{ppm})$} & $\Theta_{\text {c.m. }}(\mathrm{deg})$ & \multicolumn{2}{c|}{$A_{n}(\mathrm{ppm})$} \\
\hline & ${ }^{4} \mathrm{He}$ & ${ }^{208} \mathrm{~Pb}$ & & ${ }^{4} \mathrm{He}$ & ${ }^{208} \mathrm{~Pb}$ \\
\hline $0.5^{\circ}$ & & -0.09 & & & \\
$1.0^{\circ}$ & -0.72 & -0.33 & $11.0^{\circ}$ & -10.13 & -8.12 \\
$2.0^{\circ}$ & -1.68 & -0.91 & $12.0^{\circ}$ & -10.68 & -8.85 \\
$3.0^{\circ}$ & -2.71 & -1.57 & $13.0^{\circ}$ & -11.11 & -9.75 \\
$4.0^{\circ}$ & -3.77 & -2.31 & $14.0^{\circ}$ & -11.41 & -10.98 \\
$5.0^{\circ}$ & -4.83 & -3.10 & $15.0^{\circ}$ & -11.58 & -12.43 \\
$6.0^{\circ}$ & -5.87 & -3.97 & $16.0^{\circ}$ & -11.61 & -12.94 \\
$7.0^{\circ}$ & -6.88 & -4.93 & $17.0^{\circ}$ & -11.50 & -13.05 \\
$8.0^{\circ}$ & -7.82 & -5.94 & $18.0^{\circ}$ & -11.28 & -13.39 \\
$9.0^{\circ}$ & -8.69 & -6.82 & $19.0^{\circ}$ & -11.06 & -13.98 \\
$10.0^{\circ}$ & -9.46 & -7.48 & $20.0^{\circ}$ & -10.73 & -14.97 \\
\hline
\end{tabular}

TABLE I: Results for the analyzing power on ${ }^{4} \mathrm{He}$ for $3 \mathrm{GeV}$ beam energy and on ${ }^{208} \mathrm{~Pb}$ for $855 \mathrm{MeV}$ beam energy in forward kinematics. analyzing power is negative in both cases and is about $-10 p p m$ and $-4 p p m$, respectively. The analyzing power is relatively large. Experimentalists should take care to ensure that it does not contribute a large systematic error to the extraction of parity violating observables.

We showed that the account of Coulomb distortions to all orders in $Z \alpha_{e m}$ modifies significantly the elastic contribution to the analyzing power for ${ }^{208} \mathrm{~Pb}$. At the moment, only the leading order inelastic contribution was calculated, however it is plausible to assume that also this can be substantially modified, although not at the orderof-magnitude level, by the inclusion of the higher orders effects. This issue should be addressed in the future.

\section{Acknowledgments}

This work was supported in part by the US NSF grant PHY 0555232 (M.G.) and by DOE grant DE-FG0287ER40365 (C.J.H.)
[1] M.K. Jones et al., Phys. Rev. Lett. 84 (2000) 1398; O. Gayou et al., Phys. Rev. Lett. 88 (2002) 092301; V. Punjabi et al., Phys. Rev. C 71 (2005) 055202, Erratum-ibid. C 71 (2005) 069902.

[2] L. Andivahis et al., Phys. Rev. D 50 (1994) 5491; M.E. Christy et al., Phys. Rev. C 70 (2004) 015206; I.A. Qattan et al., Phys. Rev. Lett. 94 (2005) 142301, arXiv:nucl-ex/0410010.

[3] P.A.M. Guichon, M. Vanderhaeghen, Phys. Rev. Lett. 91, 142303 (2003)

[4] Jefferson Lab experiment E04-116, Beyond the Born approximation: a precise comparison of $e^{+} p$ and $e^{-} p$ scattering in $C L A S$, W.K. Brooks et al. spokespersons

[5] J. Arrington et al., Two-photon exchange and elastic scattering of electrons/positrons on the proton, proposal for an experiment at VEPP-3 (2004), nucl-ex/0408020

[6] S.P. Wells et al. [SAMPLE Collaboration], Phys. Rev. C 63 (2001) 064001; F. Maas et al. [MAMI A4 Collaboration], Phys. Rev. Lett. 94 (2005) 082001; SLAC E158 Experiment, contact person K. Kumar; G. Cates, K. Kumar and D. Lhuillier, spokespersons HAPPEX-2 Experiment, JLab E-99-115; D. Beck, spokesperson JLab/G0 Experiment, JLabE-00 - 6, E-01-116.

[7] A. De Rujula, J.M. Kaplan, E. De Rafael, Nucl. Phys. B 35, 365 (1971)

[8] A. Afanasev, I. Akushevich and N.P. Merenkov,
arXiv:hep-ph/0208260; B. Pasquini and M. Vanderhaeghen, Phys. Rev. C 70 (2004) 045206; L. Diaconescu and M.J. Ramsey-Musolf, Phys. Rev. C 70 (2004) 054003; M. Gorchtein, P.A.M. Guichon and M. Vanderhaeghen, Nucl Phys. A 741 (2004) 234; A.V. Afanasev, N.P. Merenkov, Phys. Lett. B 599 (2004) 48; Phys. Rev. D 70 (2004) 073002; A.V. Afanasev, N.P. Merenkov, hep-ph/0407167 v2; M. Gorchtein, hep-ph/0505022 M. Gorchtein, Phys. Rev. C 73, 055201 (2006); D. Borisyuk and A. Kobushkin, nucl-th/0508053 E.D. Cooper and C.J. Horowitz, Phys. Rev. bf C 72, 034602 (2005)

[9] Jefferson Laboratory Experiment E-00-03, Spokespersons R. Michaels, P.A. Souder, and G.M. Urcioli

[10] Jefferson Laboratory Experiment E-00-114, Spokespersons D.S. Armstrong and R. Michaels

[11] A.V. Afanasev and N.P. Merenkov, Phys. Rev. D 70, 073002 (2004); Phys. Lett. B 599, 48 (2004)

[12] M. Gorshtein, Phys. Rev. C 73, 035213 (2006); Phys. Lett. B 644, 322 (2007)

[13] T.H. Bauer, R.D. Spital, D.R. Yennie, and F.M. Pipkin, Rev. Mod. Phys. 50, 261 (1978) [Erratum - ibid. 51, 407 (1979)]

[14] E.D. Cooper and C.J. Horowitz, Phys. Rev. bf C 72, 034602 (2005)

[15] A.V. Afanasev, [arXiv: hep-ph/0711.3065] 